\title{
Interferometric electro-optical signal processors with partially coherent illumination
}

\author{
Joseph Rosen \\ Department of Electrical Engineering, Technion-Israel Institute of Technology, Haifa 32000, Israel \\ Mordechai Segev \\ California Institute of Technology, Pasadena, California 91125 \\ Joseph Shamir \\ Department of Electrical Engineering, Technion-Israel Institute of Technology, Haifa 32000, Israel
}

Amnon Yariv

California Institute of Technology, Pasadena, California 91125

Received December 11, 1991; revised manuscript received April 6, 1992; accepted April 8, 1992

\begin{abstract}
Fourier- and Hartley-related transforms are realized in a family of interferometers. The implementation of these interferometers as image correlators is investigated theoretically and experimentally with both coherent and spatially incoherent illumination. Several correlators that can be used for pattern recognition are studied
\end{abstract} and demonstrated experimentally as special cases.

\section{INTRODUCTION}

Optical image processing can derive significant benefits from the introduction of interferometric methods. The use of a shearing interferometer to yield the cosine Fourier transform of an object illuminated by spatially incoherent light is well known., ${ }^{1,2}$ Other applications of the same interferometer include the sine transform ${ }^{3-5}$ and the Hartley transform (HT) in coherent ${ }^{6,7}$ and incoherent ${ }^{8}$ illumination. In this paper we consider some additional properties of both coherent and incoherent interferometers for signal processing. Since these processing architectures usually contain Fourier transformations (FT's), we refer to them as Fourier interferometers (FI's). A generalized model of the FI is discussed in Section 2.

One important attribute of the HT is that it yields a real transform for a real input function. However, this is not necessarily the case for an arbitrary complex input function. We show in the analysis of Section 3 that system modifications are possible for generating real transforms also for complex functions.

The joint transform correlator ${ }^{9}$ (JTC) is a convenient system for obtaining the cross correlation between two functions without the need for holographic filters and complicated alignment procedures. While the conventional JTC required fully coherent illumination, we demonstrate in Section 5 that a JTC implemented by the FI can be operated under spatially incoherent illumination. In the proposed JTC the FT of the input objects is performed with incoherent illumination, but the processing of the joint spectrogram to obtain the correlation uses coherent light. The preliminary experimental results confirm the theoretical predictions.
In Section 6 we propose and demonstrate a partially incoherent correlator characterized by Fourier transforming an incoherently illuminated object, multiplying the transform by a filter, and performing an additional FT with coherent illumination. This configuration is useful in a situation in whch it is preferable to illuminate coherently the spatial frequency plane rather than to convert the input image into a coherently illuminated image.

\section{GENERALIZED MODEL OF A FOURIER INTERFEROMETER}

The schematic diagram of Fig. 1 represents a generalized FI in which two channels are illuminated by a finite extended quasi-monochromatic light source $\left(I_{s}\right)$ located at the far field relative to the input plane of the interferometer. The light distributions in the two channels are rotated with respect to each other by an angle $\phi$. Masks $g_{1}(\mathbf{r})$ and $g_{2}(\mathbf{r})$ are located in the two channels, and the far-field diffraction distributions are superposed at the output detector array. Throughout this paper we consider the far-field approximation with the understanding that this is always achievable by using lens transformations. The output signal from the detector array is time averaged during an integration time $T$. The measured intensity distribution can thus be written in the form

$$
\begin{aligned}
I(\mathbf{u})= & \frac{1}{T} \int_{-T / 2}^{T / 2} \mid \int_{\mathscr{R}} e_{1}(\mathbf{r}, t) g_{1}(\mathbf{r}) C_{1}(\mathbf{u}) \exp \left(j \frac{k}{L} \mathbf{u} \cdot \mathbf{r}\right) \\
& +\left.e_{2}(\mathbf{r}, t) g_{2}(\mathbf{r}) C_{2}(\mathbf{u}) \exp \left(j \frac{k}{L} \mathbf{u} \cdot \mathbf{r}\right) \mathrm{d} \mathbf{r}\right|^{2} \mathrm{~d} t,
\end{aligned}
$$

where $e_{i}(\mathbf{r}, t)(i=1,2)$ are the complex amplitude field dis- 


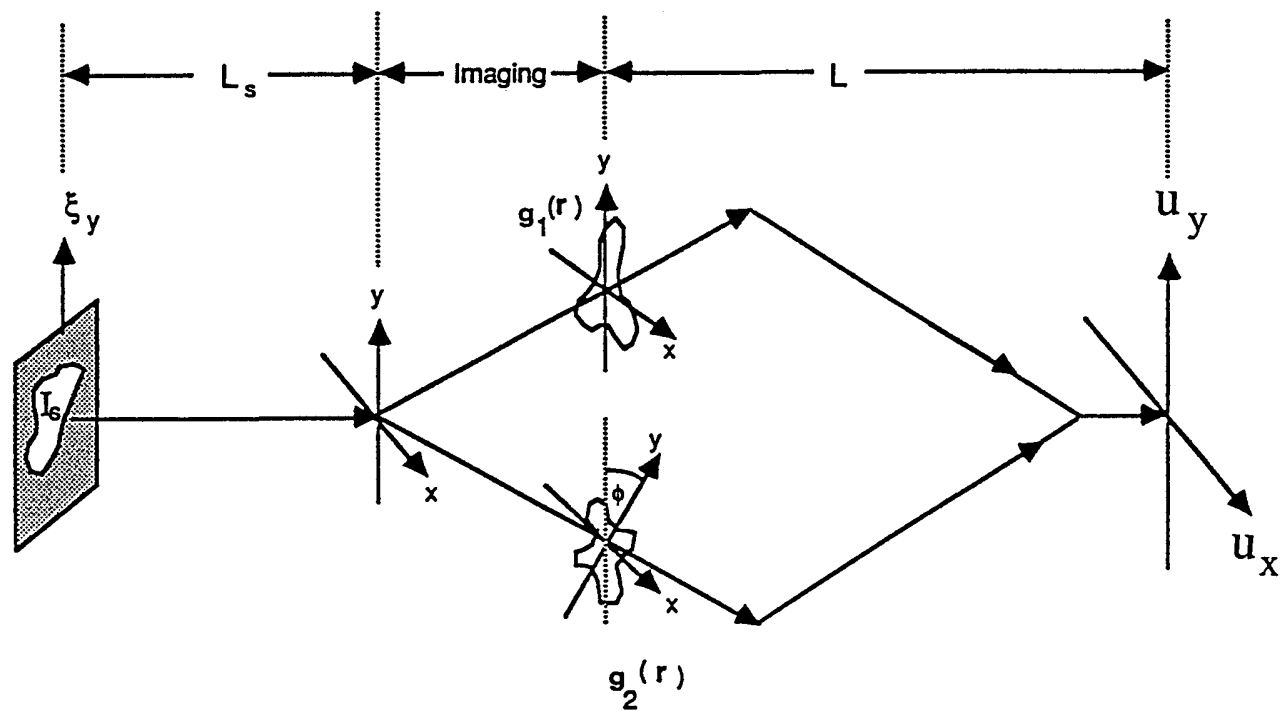

Fig. 1. Schematic illustration of the generalized model of the FI.

tributions incident upon the transparencies and $\mathbf{r}$ and $\mathbf{u}$ are the coordinate vectors of the interferometer input and output planes, respectively. The function $C_{i}(\mathbf{u})$ represents the diffraction conditions of each channel. For example, it can be a quadratic phase factor that depends on the longitudinal position of the input mask, or it may be a linear phase factor that depends on the transversal position of the input object. Alternatively $C_{i}(\mathbf{u})$ may represent a filter mask located in the $\mathbf{u}$ plane. By performing the squaring operation, we can write Eq. (1) in the form

$$
\begin{aligned}
I(\mathbf{u})= & \left|C_{1}(\mathbf{u})\right|^{2} \int_{\mathscr{R}} \int_{\mathscr{R}}\left[I_{1}(\mathbf{r}) I_{1}\left(\mathbf{r}^{\prime}\right)\right]^{1 / 2} g_{1}(\mathbf{r}) g_{1}^{*}\left(\mathbf{r}^{\prime}\right) \gamma_{11}\left(\mathbf{r}, \mathbf{r}^{\prime}\right) \\
& \times \exp \left[j \frac{k}{L} \mathbf{u} \cdot\left(\mathbf{r}-\mathbf{r}^{\prime}\right)\right] \mathrm{d} \mathbf{r} \mathrm{d} \mathbf{r}^{\prime} \\
& +\left|C_{2}(\mathbf{u})\right|^{2} \int_{\mathscr{R}} \int_{\mathscr{R}}\left[I_{2}(\mathbf{r}) I_{2}\left(\mathbf{r}^{\prime}\right)\right]^{1 / 2} g_{2}(\mathbf{r}) g_{2}{ }^{*}\left(\mathbf{r}^{\prime}\right) \gamma_{22}\left(\mathbf{r}, \mathbf{r}^{\prime}\right) \\
& \times \exp \left[j \frac{k}{L} \mathbf{u} \cdot\left(\mathbf{r}-\mathbf{r}^{\prime}\right)\right] \mathrm{d} \mathbf{r} \mathrm{d} \mathbf{r}^{\prime} \\
& +C_{1}(\mathbf{u}) C_{2}^{*}(\mathbf{u}) \int_{\mathscr{R}} \int_{\mathscr{R}}\left[I_{1}(\mathbf{r}) I_{2}\left(\mathbf{r}^{\prime}\right)\right]^{1 / 2} g_{1}(\mathbf{r}) g_{2}{ }^{*}\left(\mathbf{r}^{\prime}\right) \gamma_{12}\left(\mathbf{r}, \mathbf{r}^{\prime}\right) \\
& \times \exp \left[j \frac{k}{L} \mathbf{u} \cdot\left(\mathbf{r}-\mathbf{r}^{\prime}\right)\right] \mathrm{d} \mathbf{r d} \mathbf{r}^{\prime} \\
& +C_{1}^{*}(\mathbf{u}) C_{2}(\mathbf{u}) \int_{\mathscr{R}} \int_{\mathscr{R}}\left[I_{1}\left(\mathbf{r}^{\prime}\right) I_{2}(\mathbf{r})\right]^{1 / 2} g_{1}^{*}\left(\mathbf{r}^{\prime}\right) g_{2}(\mathbf{r}) \gamma_{21}\left(\mathbf{r}, \mathbf{r}^{\prime}\right) \\
& \times \exp \left[j \frac{k}{L} \mathbf{u} \cdot\left(\mathbf{r}-\mathbf{r}^{\prime}\right)\right] \mathrm{d} \mathbf{r d} \mathbf{r}^{\prime}
\end{aligned}
$$

where $\gamma_{i j}$ are the coherence functions defined by

$$
\begin{aligned}
\gamma_{i j}\left(\mathbf{r}, \mathbf{r}^{\prime}\right) & \equiv \frac{\left\langle e_{i}(\mathbf{r}, t), e_{j}^{*}\left(\mathbf{r}^{\prime}, t\right)\right\rangle}{\left[I_{i}(\mathbf{r}) I_{j}\left(\mathbf{r}^{\prime}\right)\right]^{1 / 2}}, \\
\left\langle e_{i}(\mathbf{r}, t), e_{j}^{*}\left(\mathbf{r}^{\prime}, t\right)\right\rangle & \equiv \frac{1}{T} \int_{-T / 2}^{T / 2} e_{i}(\mathbf{r}, t) e_{j}{ }^{*}\left(\mathbf{r}^{\prime}, t\right) \mathrm{d} t, \\
I_{i}(\mathbf{r}) & \equiv \frac{1}{T} \int_{-T / 2}^{T / 2}\left|e_{i}(\mathbf{r}, t)\right|^{2} \mathrm{~d} t .
\end{aligned}
$$

The interferometer input plane is located in the farfield region of the source; therefore, according to the Van Cittert-Zernike theorem, ${ }^{10} \gamma$ is given by the normalized inverse FT of the intensity distribution of the light source by the relation

$$
\gamma\left(\mathbf{r}, \mathbf{r}^{\prime}\right)=\frac{\exp (j \psi) \int I_{s}(\boldsymbol{\xi}) \exp \left[-j\left(2 \pi / \lambda L_{s}\right) \xi \cdot\left(\mathbf{r}-\mathbf{r}^{\prime}\right)\right] \mathrm{d} \boldsymbol{\xi}}{\int I_{s}(\boldsymbol{\xi}) \mathrm{d} \boldsymbol{\xi}},
$$

where $\xi$ is the coordinate vector of the source plane, $I_{s}(\xi)$ is the intensity distribution of the source, $L_{s}$ is the distance between the source and the object plane, and $\psi$ is defined by

$$
\psi=\frac{\pi}{\lambda L_{s}}\left(|\mathbf{r}|^{2}-\left|\mathbf{r}^{\prime}\right|^{2}\right)
$$

Since the quadratic phase factor $\exp (j \psi)$ can be canceled by properly positioned lenses, it is dropped in the analysis that follows.

To simplify Eq. (2) we assume equal and constant illumination for both channels $\left[\sqrt{I_{1}(\mathbf{r})}=\sqrt{I_{2}\left(\mathbf{r}^{\prime}\right)}=\right.$ constant $]$ and spatially stationary statistics within the apertures of interest [as is also the case according to Eq. (5)]:

$$
\begin{aligned}
& \gamma_{11}\left(\mathbf{r}, \mathbf{r}^{\prime}\right)=\gamma\left(\mathbf{r}-\mathbf{r}^{\prime}\right), \\
& \gamma_{22}\left(\mathbf{r}, \mathbf{r}^{\prime}\right)=\gamma\left[\Phi\left(\mathbf{r}-\mathbf{r}^{\prime}\right)\right] \\
& \gamma_{12}\left(\mathbf{r}, \mathbf{r}^{\prime}\right)=\gamma\left(\mathbf{r}-\Phi \mathbf{r}^{\prime}\right), \\
& \gamma_{21}\left(\mathbf{r}, \mathbf{r}^{\prime}\right)=\gamma\left(\Phi \mathbf{r}-\mathbf{r}^{\prime}\right),
\end{aligned}
$$

where $\Phi$ is the rotation matrix representing the relative rotation angle of the two channels, $\phi$. Under these assumptions, Eq. (2) becomes 


$$
\begin{aligned}
I(\mathbf{u})= & \left|C_{1}(\mathbf{u})\right|^{2} \int_{\mathscr{R}} \int_{\mathscr{R}} g_{1}(\mathbf{r}) g_{1}^{*}\left(\mathbf{r}^{\prime}\right) \gamma\left(\mathbf{r}-\mathbf{r}^{\prime}\right) \\
& \times \exp \left[j \frac{k}{L} \mathbf{u} \cdot\left(\mathbf{r}-\mathbf{r}^{\prime}\right)\right] \mathrm{d} \mathbf{r} \mathrm{d} \mathbf{r}^{\prime} \\
& +\left|C_{2}(\mathbf{u})\right|^{2} \int_{\mathscr{R}} \int_{\mathscr{R}} g_{2}(\mathbf{r}) g_{2}{ }^{*}\left(\mathbf{r}^{\prime}\right) \gamma\left[\Phi\left(\mathbf{r}-\mathbf{r}^{\prime}\right)\right] \\
& \times \exp \left[j \frac{k}{L} \mathbf{u} \cdot\left(\mathbf{r}-\mathbf{r}^{\prime}\right)\right] \mathrm{d} \mathbf{r} \mathrm{d} \mathbf{r}^{\prime} \\
& +W(\mathbf{u}) \int_{\mathscr{R}} \int_{\mathscr{R}} g_{1}(\mathbf{r}) g_{2}{ }^{*}\left(\mathbf{r}^{\prime}\right) \gamma\left(\mathbf{r}-\Phi \mathbf{r}^{\prime}\right) \\
& \times \exp \left[j \frac{k}{L} \mathbf{u} \cdot\left(\mathbf{r}-\mathbf{r}^{\prime}\right)\right] \mathrm{d} \mathbf{r} \mathrm{d} \mathbf{r}^{\prime} \\
& +W^{*}(\mathbf{u}) \int_{\mathscr{R}} \int_{\mathscr{R}} g_{1}^{*}\left(\mathbf{r}^{\prime}\right) g_{2}(\mathbf{r}) \gamma\left(\Phi \mathbf{r}-\mathbf{r}^{\prime}\right) \\
& \times \exp \left[j \frac{k}{L} \mathbf{u} \cdot\left(\mathbf{r}-\mathbf{r}^{\prime}\right)\right] \mathrm{d} \mathbf{r} \mathrm{d} \mathbf{r}^{\prime},
\end{aligned}
$$

where $W(\mathbf{u})=C_{1}(\mathbf{u}) C_{2}{ }^{*}(\mathbf{u})$. In Section 3 we consider the special case of coherent illumination, and in Section 4 we consider this intensity distribution as a holographic recording with a partially coherent illumination.

\section{FOURIER INTERFEROMETRY AND THE MODIFIED HARTLEY TRANSFORM}

In this section we consider the implementation of the FI for extending the HT. According to Refs. 6, 7, and 11, the HT is defined such that it yields a real transform if the input function is real. To obtain a real transform distribution even when the input is an arbitrary complex function, we extend the definition and consider its optical realization.

The HT of a real function $g(\mathbf{r})$ can be obtained by the FI under coherent ${ }^{6}$ or incoherent ${ }^{3}$ illumination. In both cases we have to substitute into Eq. (8) the following functions: $\quad C_{1}(\mathbf{u})=\exp (j \alpha / 2), C_{2}(\mathbf{u})=\exp (-j \alpha / 2)(0<$ $\alpha<\pi)$, and $g_{1}(\mathbf{r})=g_{2}(-\mathbf{r})=g(\mathbf{r})$, and for the incoherent case it is required that $\phi=180^{\circ}$. When the system is coherently illuminated $\gamma_{i j}\left(\mathbf{r}, \mathbf{r}^{\prime}\right)=$ constant, and the complex amplitude distribution in the spatial frequency domain $\mathbf{f}_{r}\left(\mathbf{f}_{r}=\mathbf{u} / \lambda L\right)$ becomes

$$
E_{H}\left(\mathbf{f}_{r}\right) \propto \exp \left(j \frac{\alpha}{2}\right) G\left(\mathbf{f}_{r}\right)+\exp \left(-j \frac{\alpha}{2}\right) G\left(-\mathbf{f}_{r}\right)
$$

where $G\left(\mathbf{f}_{r}\right)$ is the FT of $g(\mathbf{r})$ and we assumed that the output plane coincides with the FT plane of both channels.

The constant phase factors $\exp ( \pm j \alpha / 2)$ may be obtained from the optical-path difference between the two channels. When $\alpha$ is equal to 0 or $\pi, E_{H}\left(\mathbf{f}_{r}\right)$ is proportional to the cosine FT or the sine transform of $g(\mathbf{r})$, respectively. When $\alpha=\pi / 2$ it is proportional to the HT, and when $0<\alpha<\pi$ it is considered the generalized $\mathrm{HT}^{11}$ (GHT). Unlike the FT, all these transforms are real functions if $g(\mathbf{r})$ is real. However, only the GHT preserves all the complex information of $G\left(\mathbf{f}_{r}\right)$.
If the illumination is completely incoherent, we have $\gamma\left(\mathbf{r}-\mathbf{r}^{\prime}\right)=\delta\left(\mathbf{r}-\mathbf{r}^{\prime}\right)$, which substituted into Eq. (8) yields

$$
\begin{gathered}
I_{H}\left(\mathbf{f}_{r}\right) \propto 2 \int_{\mathscr{R}}|g(\mathbf{r})|^{2} \mathrm{~d} \mathbf{r}+\exp (j \alpha) \tilde{G}\left(2 \mathbf{f}_{r}\right) \\
+\exp (-j \alpha) \tilde{G}\left(-2 \mathbf{f}_{r}\right),
\end{gathered}
$$

where $\tilde{G}\left(\mathbf{f}_{r}\right)$ is the FT of $|g(\mathbf{r})|^{2}$. Once again a GHT is obtained, except that this time it is the transform of the intensity of the input transparency with an additional bias term rather than the transform of the complex amplitude. A different incoherent $\mathrm{HT}$ of a function $g(\mathbf{r})$ can be obtained if a constant distribution $A$ is introduced in one channel while $g(\mathbf{r})$ is displayed in the other. The HT of $g(\mathbf{r})$ in this case is

$$
\begin{aligned}
I_{H^{\prime}}\left(\mathbf{f}_{r}\right) \propto & \int_{\mathscr{R}}|g(\mathbf{r})|^{2} \mathrm{~d} \mathbf{r}+\int_{\mathscr{R}}|A|^{2} \mathrm{~d} \mathbf{r}+A^{*} \exp (j \alpha) G\left(2 \mathbf{f}_{r}\right) \\
& +A \exp (-j \alpha) G^{*}\left(2 \mathbf{f}_{r}\right) .
\end{aligned}
$$

In real-world applications the input function is not always a real one. When a coherent plane wave is reflected from a nonplanar object or when it passes through a phasemodulating medium, the light may be characterized by a nonplanar phase front. Hence the field distribution is expressed by a two-dimensional complex function. If the goal is to find a transform that yields, for any input function, a real transformed function, the HT is not sufficient. To obtain a real transform for a complex function also, we define a modified HT and call it the real HT (RHT), since it generates a real transformed function for any complex input. The formal definition of the two-dimensional RHT of a complex function $g(\mathbf{r})=|g(\mathbf{r})| \exp [j \Phi(\mathbf{r})]$ is taken as

$$
H_{\alpha}\left(\mathbf{f}_{r}\right)=\sqrt{2} \int \lg (\mathbf{r}) \mid \cos \left[2 \pi \mathbf{f}_{r} \cdot \mathbf{r}+\Phi(\mathbf{r})+\alpha / 2\right] \mathrm{d} \mathbf{r},
$$

where $0<\alpha<\pi$. The RHT is identical to the GHT [that is, $\left.H_{\alpha}\left(\mathbf{f}_{r}\right)=E_{H}\left(\mathbf{f}_{r}\right)\right]$ when $\Phi(\mathbf{r})=0$ or $\pi$, i.e., when $g(\mathbf{r})$ is real.

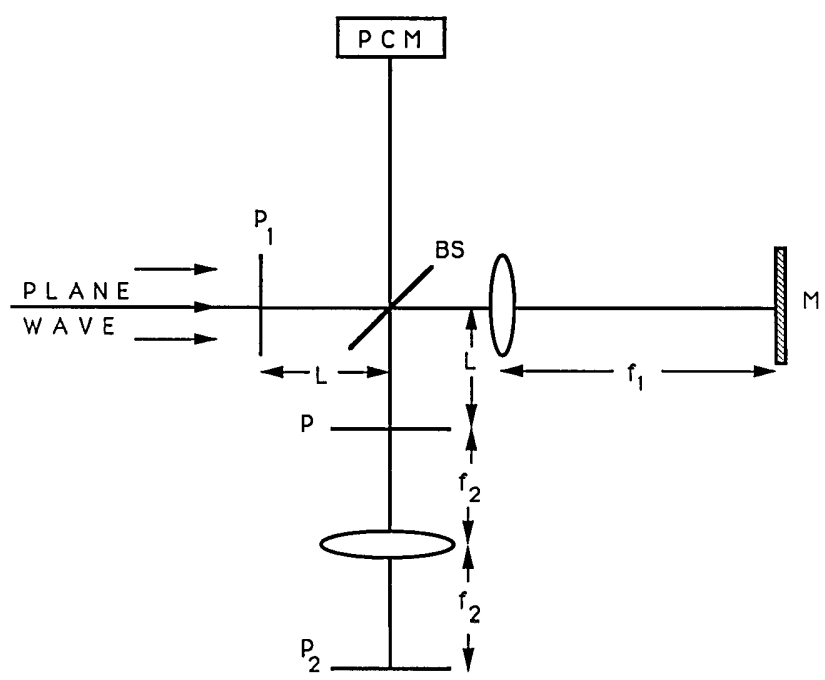

Fig. 2. Shearing interferometer, as assigned to perform the RHT. 


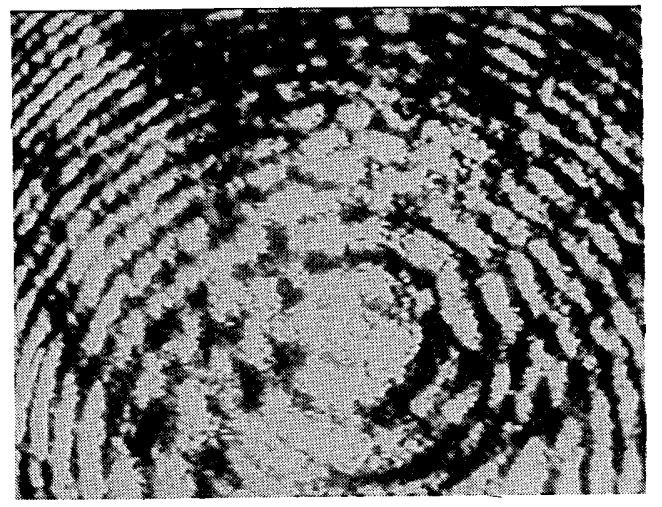

Fig. 3. Experimental results for the modified HT obtained with the interferometer of Fig. 2.

The optical implementation of the RHT with the system of Fig. 2 becomes evident if Eq. (12) is rewritten in the form

$$
\begin{aligned}
H_{\alpha}\left(\mathbf{f}_{r}\right)= & \frac{1}{\sqrt{2}} \exp \left(j \frac{\alpha}{2}\right) \int g(\mathbf{r}) \exp \left(j 2 \pi \mathbf{f}_{r} \cdot \mathbf{r}\right) \mathrm{d} \mathbf{r} \\
& +\frac{1}{\sqrt{2}} \exp \left(-j \frac{\alpha}{2}\right) \int g^{*}(\mathbf{r}) \exp \left(-j 2 \pi \mathbf{f}_{r} \cdot \mathbf{r}\right) \mathrm{d} \mathbf{r} \\
= & \frac{1}{\sqrt{2}} \exp \left(j \frac{\alpha}{2}\right)\left[G\left(\mathbf{f}_{r}\right)+\exp (-j \alpha) G^{*}\left(\mathbf{f}_{r}\right)\right]
\end{aligned}
$$

The optical system differs from that used to perform an ordinary optical $\mathrm{HT}^{6}$ by a phase-conjugate mirror (PCM) that replaces a regular mirror in one of the channels. This channel reflects the function $g^{*}(\mathbf{r})$, while the second channel reflects the function $g(-\mathbf{r})$ if the input function is $g(\mathbf{r})$. The FT of the two functions is performed by the lens located at the common channel (before plane $\mathrm{P}_{2}$ in Fig. 2). The result is given by Eq. (13) when the coordinate axes $\mathrm{P}_{2}$ are inverted.

As mentioned above, the resulting function in the output plane $\mathrm{P}_{2}$ is always real. However, it does not always preserve all the information contained in the input function. It is reasonable that an arbitrary complex function cannot be compressed into a real one without information loss. It works for a real input function because of its Hermitian property, which is not possessed by an arbitrary complex function. Nevertheless, if we also have the complementary distribution $\tilde{H}_{\alpha+\pi}\left(\mathbf{f}_{r}\right)$ it is possible to reconstruct the original input function by using the relation

$$
\begin{aligned}
g(\mathbf{r})= & \frac{\exp [j(\alpha / 2)]}{2} \int\left[\tilde{H}_{\alpha}\left(\mathbf{f}_{r}\right)+\tilde{H}_{\alpha+\pi}\left(\mathbf{f}_{r}\right)\right] \\
& \times \exp \left(-j 2 \pi \mathbf{f}_{r} \cdot \mathbf{r}\right) \mathrm{d} \mathbf{f}_{r} .
\end{aligned}
$$

The complementary distribution $\tilde{H}_{\alpha+\pi}\left(\mathbf{f}_{r}\right)$ can be obtained by using a backpropagating reflection from a second beam splitter located between the $\mathrm{P}_{1}$ plane and beam splitter BS of Fig. 2. As is pointed out in Refs. 12 and 13, the use of a PCM in an interferometric configuration has the advantage of self-referencing and phase-sensitivity enhancement.

Experimental Demonstration of the Modified Hartley Transform. The results of an experimental demonstration of the modified HT are shown in Fig. 3. We chose to transform a phase element of which the modified HT (the RHT) significantly differs from the ordinary HT. In our case the RHT yields a real function in the transform plane instead of a complex one (in the HT case). In this experiment the system shown in Fig. 2 is illuminated by a coherent plane wave from an Ar laser. Our object was a lens, followed by an off-axis aperture (this gives an off-axis quadratic phase as an input object), both located at the $P_{1}$ plane of Fig. 2. The PCM was realized by a photorefractive oscillator (semilinear passive $\mathrm{PCM}^{14}$ ) using a $\mathrm{BaTiO}_{3}$ crystal. The conjugated and the inverted images were obtained at plane $\mathrm{P}$ located at a distance $L$ from beam splitter BS ( $L$ is identical to the distance between $P_{1}$ and BS), and the RHT appeared at the output $\mathrm{P}_{2}$ plane. The RHT's of the off-centered section of the quadratic phase front (our input) are the off-axis circular interference fringes shown in Fig. 3. The optical axis is located at the bright spot in the middle of Fig. 3 and does not coincide with the center of the concentric interference fringes. The gaps and the noise in the rings are due to interference effects occasionally obtained in coherent systems. In the ordinary HT the circular interference fringes become straight lines. ${ }^{6}$ Since the signs of the quadratic phases are the same in both channels, taking the intensity of their interference distribution results in the loss of all the information regarding the quadratic phase in the HT case. As demonstrated in Fig. 3, our RHT manifests the quadratic phase information in a real function.

\section{GENERAL MODEL FOR THE HOLOGRAM RECONSTRUCTION}

In this section we assume that the intensity distribution given by Eq. (8) is recorded as a transparency. This transparency can be treated as a FT hologram to be reconstructed with coherent illumination in a conventional 2 - $f$ optical system. The output distribution $c(\tilde{\mathbf{r}})$ at the back focal plane of the reconstruction lens is the FT of $I(\mathbf{u})$ given by Eq. (8):

$$
\begin{aligned}
c(\tilde{\mathbf{r}})= & \mathscr{F}\left\{\left|C_{1}(\mathbf{u})\right|^{2}\right\} * \gamma(\tilde{\mathbf{r}}) \int_{\mathscr{R}} g_{1}(\mathbf{r}) g_{1} *(\mathbf{r}-\tilde{\mathbf{r}}) \mathrm{d} \mathbf{r} \\
& +\mathscr{F}\left\{\left|C_{2}(\mathbf{u})\right|^{2}\right\} * \gamma(\Phi \tilde{\mathbf{r}}) \int_{\mathscr{R}} g_{2}(\mathbf{r}) g_{2} *(\mathbf{r}-\tilde{\mathbf{r}}) \mathrm{d} \mathbf{r} \\
& +\mathscr{F}\{W(\mathbf{u})\} * \int_{\mathscr{R}} g_{1}(\mathbf{r}) g_{2}{ }^{*}(\mathbf{r}-\tilde{\mathbf{r}}) \gamma(\mathbf{r}-\Phi \mathbf{r}+\Phi \tilde{\mathbf{r}}) \mathrm{d} \mathbf{r} \\
& +\mathscr{F}_{\mathscr{F}}\left\{W^{*}(\mathbf{u})\right\} * \int_{\mathscr{R}} g_{2}(\mathbf{r}) g_{1} *(\mathbf{r}-\tilde{\mathbf{r}}) \gamma(\Phi \mathbf{r}-\mathbf{r}+\tilde{\mathbf{r}}) \mathrm{d} \mathbf{r},
\end{aligned}
$$

where $*$ denotes the convolution operation. The first two terms, located around the origin, cannot be separated from each other and thus are of no interest here. We are concerned mainly with the other two terms, which can be spatially isolated by using the convolution with the FT of $W(\mathbf{u})$ and $W^{*}(\mathbf{u})$.

Equation (15) is a generalized expression of which some special cases are well known. For example, if the source is coherent and $W(\mathbf{u})$ is a linear phase factor, then the third and the fourth terms yield the cross correlation between $g_{1}(\mathbf{r})$ and $g_{2}(\mathbf{r})$. This is the conventional JTC. ${ }^{9}$ 


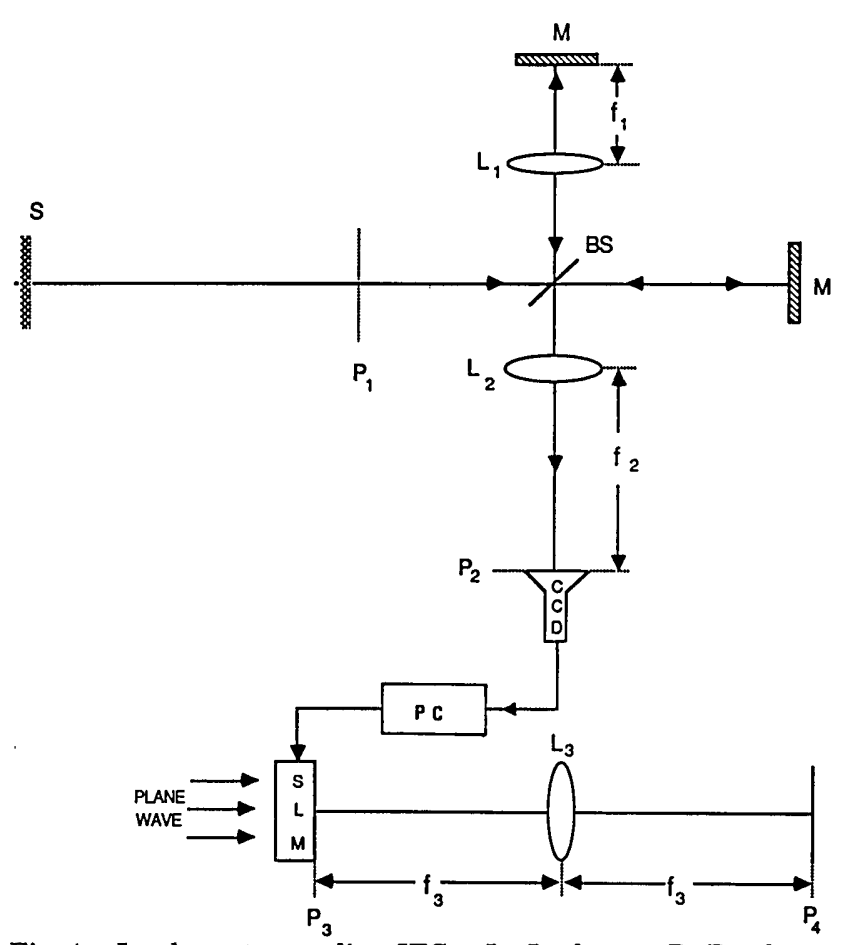

Fig. 4. Incoherent-recording JTC: $\mathrm{L}_{1}-\mathrm{L}_{3}$, lenses; $\mathrm{P}_{1}-\mathrm{P}_{4}$, planes; M's, mirrors; S, light source; CCD, charge-coupled device; PC, personal computer; BS, beam splitter.

An unconventional processor may be obtained by taking $\phi=180^{\circ}$. For this choice the third term of Eq. (15), for example, is given by

$$
s(\tilde{\mathbf{r}})=\int_{\mathscr{R}} g_{1}(\mathbf{r}) g_{2}^{*}(\mathbf{r}-\tilde{\mathbf{r}}) \gamma(2 \mathbf{r}-\tilde{\mathbf{r}}) \mathrm{d} \mathbf{r} .
$$

This unconventional expression may have various applications in image processing by the substitution of special functions. Some of these are still under investigation, while in the following sections we consider two special kinds of correlator operated under incoherent illumination.

\section{INCOHERENT JOINT TRANSFORM CORRELATOR}

To implement a JTC we use the well-known rotational shearing interferometer ${ }^{1,2,4,5}$ in the configuration proposed in Ref. 7 to perform a HT. Lens $\mathrm{L}_{1}$ in our system (Fig. 4) has two purposes. It rotates the input function by $180^{\circ}$ and performs imaging. Thus, if the interferometer arms have equal physical lengths, the effective diffraction paths $d_{1}$ and $d_{2}$ (measured to the entrance pupil of lens $L_{2}$ ) in the two channels differ by $4 f_{1}$, where $f_{1}$ is the focal length of the lens. Hence the two diffraction patterns superposed upon plane $\mathrm{P}_{2}$ are multiplied by two different quadratic phase factors. Defining the quadratic phase factor as

$$
\mathscr{Q}[d] \equiv \exp \left(j \frac{\pi}{\lambda} d|\mathbf{u}|^{2}\right),
$$

we obtain the intensity at plane $\mathrm{P}_{2}$ by substituting $C_{i}(\mathbf{u})=$ $2\left[1 / f_{2}\left(1-d_{i} / f_{2}\right)\right], \phi=180^{\circ}$, and $g_{1}(\mathbf{r})=g_{2}(-\mathbf{r})=g(\mathbf{r})$ into Eq. (8). If the illumination is spatially incoherent we also take $\gamma\left(\mathbf{r}-\mathbf{r}^{\prime}\right)=\delta\left(\mathbf{r}-\mathbf{r}^{\prime}\right)$, and Eq. (8) reduces to

$$
I_{2}\left(\mathbf{f}_{r}\right)=2 I_{\alpha}+2\left[\frac{4 f_{1}}{f_{2}^{2}}\right] \tilde{G}\left(\mathbf{f}_{r}\right)+2\left[-\frac{4 f_{1}}{f_{2}^{2}}\right] \tilde{G}^{*}\left(\mathbf{f}_{r}\right),
$$

where $\tilde{G}\left(\mathbf{f}_{r}\right)$ is the FT of $|g(\mathbf{r})|^{2}, f_{1}$ and $f_{2}$ are the focal lengths of lenses $\mathrm{L}_{1}$ and $\mathrm{L}_{2}$, respectively, $\mathbf{f}_{r}=2 \mathbf{u} / \lambda f_{2}$, and

$$
I_{\alpha}=\int_{\mathscr{R}}|g(\mathbf{r})|^{2} \mathrm{~d} \mathbf{r}
$$

$I_{2}\left(f_{r}\right)$ is the intensity distribution as recorded by a camera and is manipulated as follows: The first term of $I_{2}\left(f_{r}\right)$ (a dc term) is subtracted from $I_{2}\left(\mathbf{f}_{r}\right)$ to improve the efficiency. The remaining two terms are squared (electronically) and displayed on a spatial light modulator (SLM). The transmittance of the SLM is thus given by

$$
\begin{aligned}
T_{2}\left(\mathbf{f}_{r}\right)= & 2\left[\frac{8 f_{1}}{f_{2}{ }^{2}}\right]\left[\tilde{G}\left(\mathbf{f}_{r}\right)\right]^{2}+2\left[-\frac{8 f_{1}}{f_{2}{ }^{2}}\right]\left[\tilde{G}^{*}\left(\mathbf{f}_{r}\right)\right]^{2} \\
& +2 \tilde{G}\left(\mathbf{f}_{r}\right) \tilde{G}^{*}\left(\mathbf{f}_{r}\right) .
\end{aligned}
$$

The SLM is illuminated by a coherent plane wave as in the case of the conventional JTC. The FT of the third term of Eq. (20) is

$$
c(\tilde{\mathbf{r}})=\left|g\left(\frac{f_{2}}{2 f_{3}} \tilde{\mathbf{r}}\right)\right|^{2} *\left|g\left(\frac{f_{2}}{2 f_{3}} \tilde{\mathbf{r}}\right)\right|^{2}
$$

where * denotes the correlation operation that is obtained at the back focal plane of lens $L_{3}$. The other two terms are focused at distances $f_{3} \pm 8 f_{1} f_{3}{ }^{2} / f_{2}{ }^{2}$ from lens $L_{3}$. With proper parameter selection these terms contribute only a low-level diffused illumination at the back focal plane.

Using the different quadratic phase factors provided a means of separating the various terms along the $z$ axis. An alternative approach for separating the terms is the multiplication by a linear phase factor,

$$
\mathscr{L}[\mathbf{r}]=\exp \left(j \frac{\pi}{\lambda} \mathbf{r} \cdot \mathbf{u}\right),
$$

which can be obtained simply by shifting $g(\mathbf{r})$ at the input plane away from the origin. When the input function is shifted by a distance $\mathbf{r}_{d}$ and there is no diffraction path difference between the two channels, the intensity distribution $I_{2}\left(f_{r}\right)$ of Eq. (18) is replaced by

$$
I_{\mathscr{L}}\left(\mathbf{f}_{r}\right)=2 I_{\alpha}+\mathscr{L}\left[\frac{4 \mathbf{r}_{d}}{f_{2}}\right] \tilde{G}\left(\mathbf{f}_{r}\right)+\mathscr{L}\left[-\frac{4 \mathbf{r}_{d}}{f_{2}}\right] \tilde{G}^{*}\left(\mathbf{f}_{r}\right) .
$$

The displayed distribution on the SLM becomes

$$
\begin{aligned}
T_{\mathscr{L}}\left(\mathbf{f}_{r}\right)= & \mathscr{L}\left[\frac{8 \mathbf{r}_{d}}{f_{2}}\right]\left[\tilde{G}\left(\mathbf{f}_{r}\right)\right]^{2}+\mathscr{L}\left[-\frac{8 \mathbf{r}_{d}}{f_{2}}\right]\left[\tilde{G}^{*}\left(\mathbf{f}_{r}\right)\right]^{2} \\
& +2 \tilde{G}\left(\mathbf{f}_{r}\right) \tilde{G}^{*}\left(\mathbf{f}_{r}\right) .
\end{aligned}
$$

In this case the separation of the desired correlation term of Eq. (21) is done transversally, i.e., it is obtained on axis, while the two unwanted terms appear in the 1st and -1st diffraction orders and can be easily blocked. 


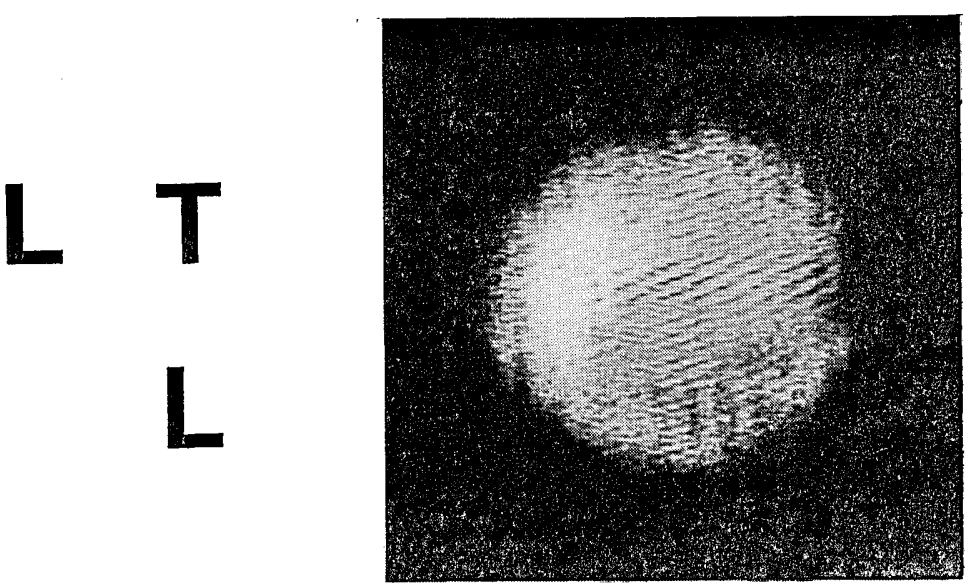

(a)

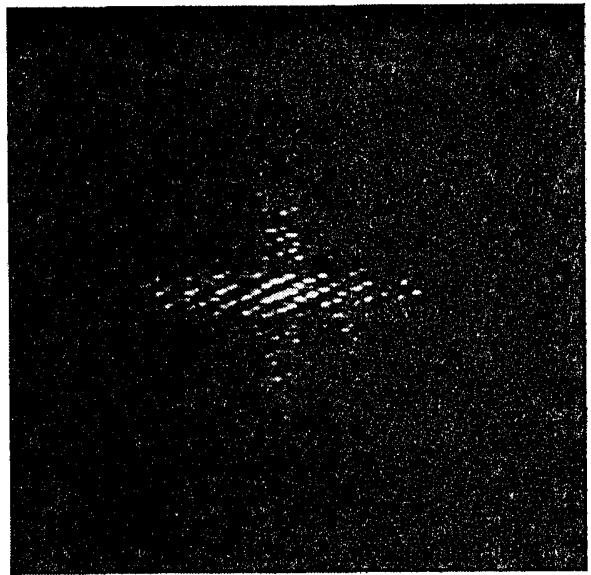

(d)

(b)

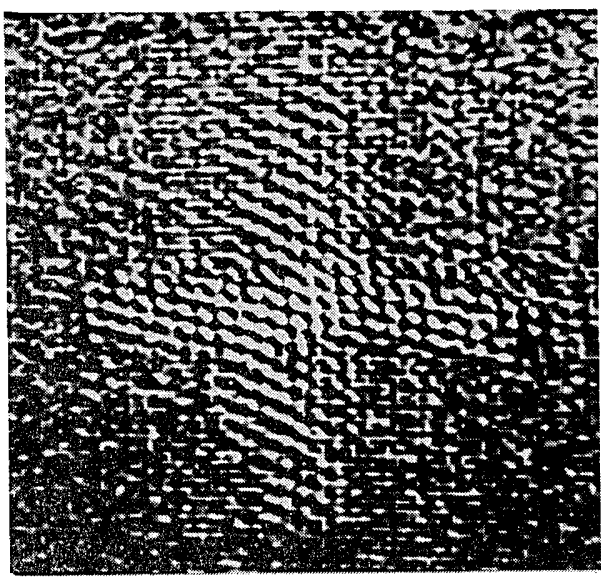

(c)

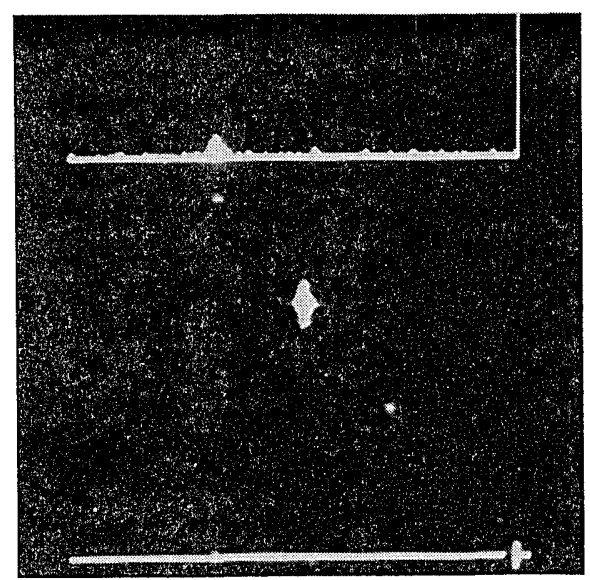

(e)

Fig. 5. (a) Input mask to the incoherent-recording JTC. The two upper letters are the tested objects, and the lower letter is the reference. (b) Intensity distribution as recorded in plane $\mathrm{P}_{2}$ of Fig. 4 when the input was the mask of (a). (c) Pattern of (b) after the bias term was subtracted. (d) Transmissivity of the SLM in Fig. 4 [the distribution shown in (c), squared]. (e) Correlation results with a cross section from right to left through the upper peak.

The proper choice between the two separation methods depends on the nature of the processed objects. To avoid overlapping between the different terms in the transverse separation, the diameter of the optical system $D$ should satisfy $D \geq 3 W_{m}$, where $W_{m} \times W_{m}$ is the area of the input image. In the longitudinal separation the limitation stems from the bandwidth of the input object. To avoid information loss, the system diameter should satisfy $D \geq$ $W_{m}+2 \lambda \Delta_{d} B_{0}$, where $\Delta_{d}$ is the longitudinal separation between the two channels and $B_{0}$ is the bandwidth of the input mask. Therefore, when the bandwidth is greater then $W_{m} / \lambda \Delta_{d}$, the transverse separation is preferred.

In both configurations a correlation between an input function $f(\mathbf{r})$ and a reference function $r(\mathbf{r})$ can be obtained by introducing both of the functions on the input mask in the form

$$
|g(\mathbf{r})|^{2}=f\left(\mathbf{r}-\mathbf{r}_{e}\right)+r\left(\mathbf{r}+\mathbf{r}_{e}\right),
$$

where $r(\mathbf{r})$ and $f(\mathbf{r})$ are spatially bounded. The autocorrelation of Eq. (21) leads to the distribution

$$
\begin{aligned}
c(\overline{\mathbf{r}})= & f(\overline{\mathbf{r}}) * f(\overline{\mathbf{r}})+r(\overline{\mathbf{r}}) * r(\overline{\mathbf{r}}) \\
& +f\left(\overline{\mathbf{r}}-2 \mathbf{r}_{e}\right) * r(\overline{\mathbf{r}})+r\left(\overline{\mathbf{r}}+2 \mathbf{r}_{e}\right) * f(\overline{\mathbf{r}}),
\end{aligned}
$$

where $\overline{\mathbf{r}}=\left(f_{2} / 2 f_{3}\right) \tilde{\mathbf{r}}$. The third term, obtained at $\overline{\mathbf{r}}=2 \mathbf{r}_{e}$, and the fourth term, obtained at $\overline{\mathbf{r}}=-2 \mathbf{r}_{e}$, are the required cross-correlation functions between $f(\mathbf{r})$ and $r(\mathbf{r})$.

\section{A. Experimental Demonstration of the Incoherent Joint Transform Correlator}

For the preliminary demonstration experiments with the incoherent JTC we used a system similar to that shown in Fig. 4 with a rotating diffuser illuminated by a laser as the light source. ${ }^{15}$ In the first experiment the input mask contained the three letters shown in Fig. 5(a), where the lower $\mathrm{L}$ is considered the reference function $r(\mathbf{r})$ and the two upper letters are the test objects $f(\mathbf{r})$. This experiment demonstrates a pattern recognition system, and thus we expect a detectable correlation peak at a position corresponding to the letter $\mathrm{L}$ on the upper line. In this experiment two identical diffraction paths were obtained by imaging the input mask in both channels to the same plane in front of lens $L_{2}$. The input mask is shifted away from the origin of the input plane to generate a linear phase. The spectrogram $I_{\mathscr{L}}(\mathbf{u})$ recorded by the CCD camera at plane $\mathrm{P}_{2}$ is shown in Fig. 5(b). The dc level is measured by blocking one channel and is subtracted from $I_{\mathscr{L}}(\mathbf{u})$. The resulting grating is depicted in Fig. 5(c). This grat- 


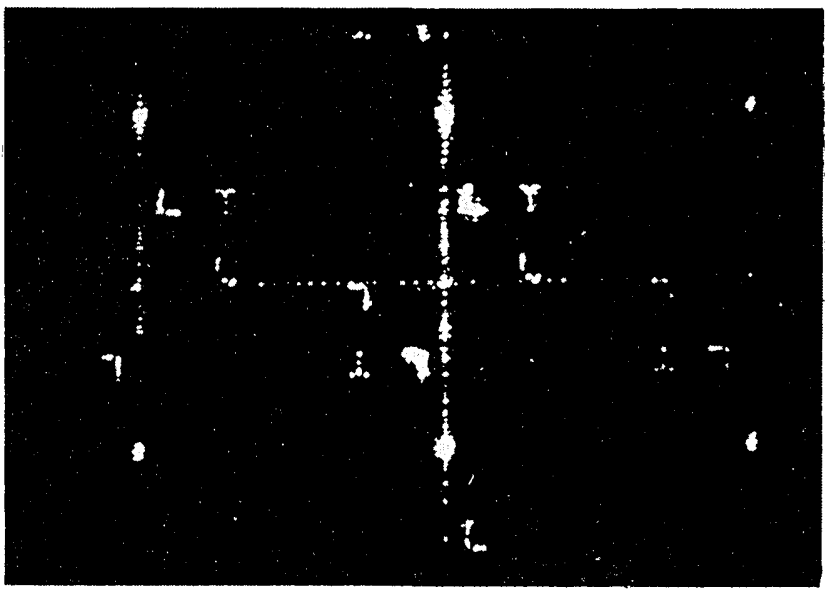

Fig. 6. Reconstruction of the grating shown in Fig. 5(b) (after binarization) obtained by coherent optical FT.

ing is squared, and the result, $T_{\mathscr{L}}(\mathbf{u})$, is shown in Fig. 5(d). Finally, $T_{\mathscr{L}}(\mathbf{u})$ is displayed on the SLM and Fourier transformed by an ordinary coherent system. The correlation results with two detected peaks at the locations of the autocorrelations of the letters $\mathrm{L}$ (at the \pm 1 st diffraction orders) are shown in Fig. 5(e).
The required transversal separation may be visualized by displaying $I_{\mathscr{L}}(\mathbf{u})$, the grating that is shown in Fig. 5(b), on the SLM and performing a coherent FT by a single lens. From the reconstructed image shown in Fig. 6, we note that the distance between $I(\mathbf{r})$ and $I(-\mathbf{r})$ fulfills the desired condition, i.e., $\mathbf{r}_{d}>W_{m} / 2$.

In the second experiment the reference function was the letter T shown in Fig. 7(a), and we used a difference in the diffraction path lengths between the two channels to implement the required separation. The effect of the resulting quadratic phase is evident from the reconstruction of the spectrogram, $I_{2}$ (u) [Fig. 7(b)] shown in Fig. 7(c). At the plane where one image is focused, its rotated twin is smeared. The correlation result is presented in Fig. 7(d), where the peaks are located at the positions of the letter $\mathrm{T}$. The correlation plane is noisier than the previous one, shown in Fig. 5(e), because the separation here is along the $z$ axis instead of the transversal plane. However, the correlation peak is still detectable above the noise level.

\section{B. Complex Reference in Incoherent Joint Transform Correlator}

The function $|g(\mathbf{r})|^{2}$ represents the intensity of the light originating from the input transparency; thus both functions $f(\mathbf{r})$ and $r(\mathbf{r})$ are positive. For the realization of a

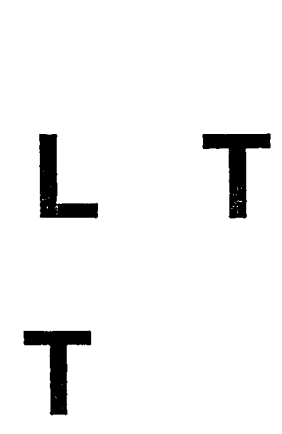

(a)

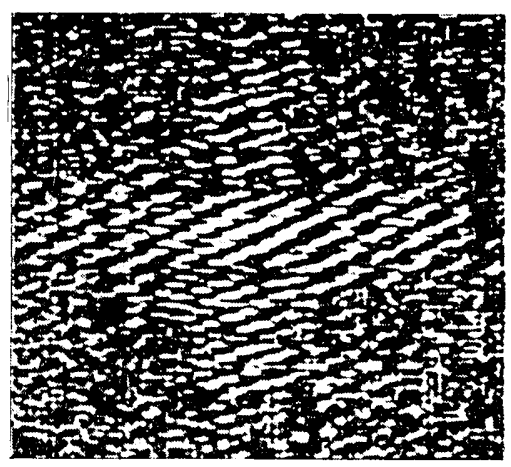

(b)

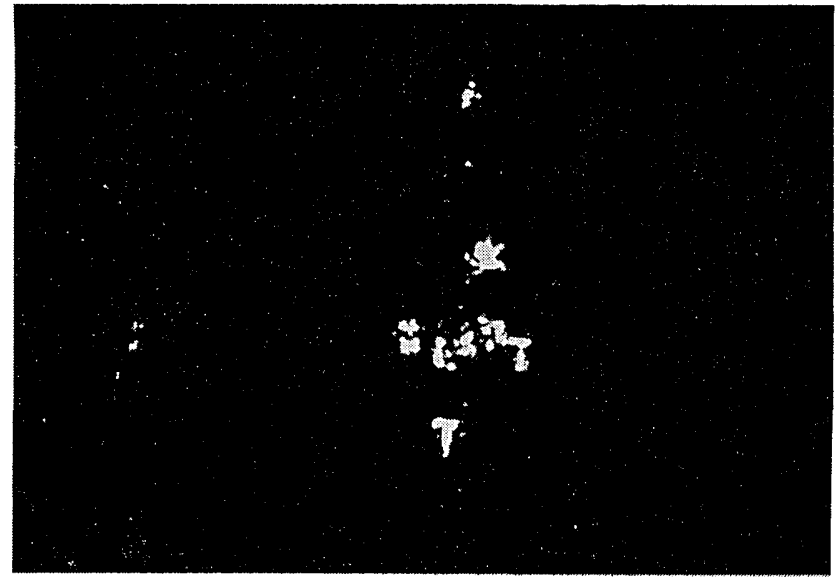

(c)

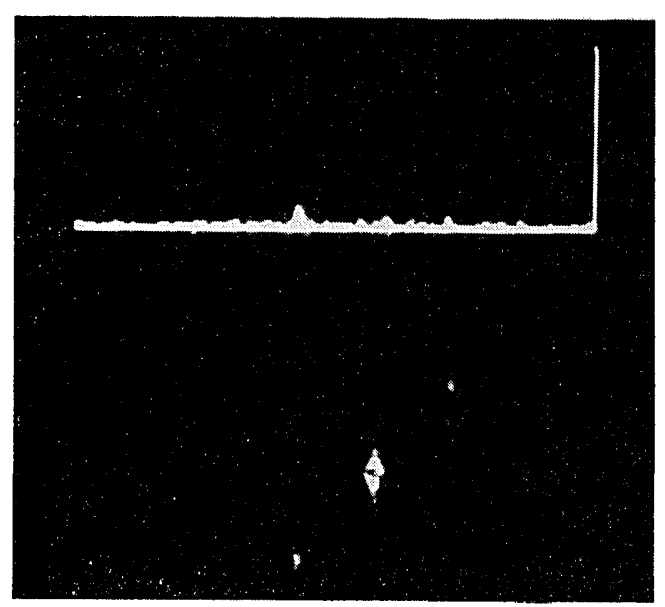

(d)

Fig. 7. (a) Input mask to the incoherent-recording JTC for the second experiment. (b) Intensity distribution as recorded in plane $\mathrm{P}_{2}$ of Fig. 4 (without the dc term) with the input mask of (a). (c) Reconstruction of the grating shown in (b) (after binarization) obtained by coherent optical FT. (d) Correlation results with a cross section from right to left through the lower peak. 
complex reference function, $f(\mathbf{r})$ and $r(\mathbf{r})$ must be coded on a carrier frequency. ${ }^{16,17}$ Let $\tilde{r}(\mathbf{r})$ be the required complex reference function with $\tilde{R}\left(\mathbf{f}_{r}\right)$ its $\mathrm{FT}$, assumed to be band limited with dimensions $W_{\tilde{R}} \times W_{\tilde{R}}$. A possible way to obtain the desired $\tilde{r}(\mathbf{r})$ is by shifting $\tilde{R}\left(\mathbf{f}_{r}\right)$ a distance $\mathbf{f}_{0}\left(\left|\mathbf{f}_{0}\right|>\right.$ $\left.W_{\tilde{R}} / 2\right)$ in the frequency domain and combining it with the conjugate version, $\tilde{R}^{*}\left(-\mathbf{f}_{r}\right)$ positioned at a distance $-\mathbf{f}_{0}$. This combination satisfies the Hermitian symmetric property, and thus the overall inverse FT, i.e., $r(\mathbf{r})$, is a real function. To make it positive also, one may add a zeroorder term at the origin of the $\left(\mathbf{f}_{r}\right)$ domain, so that the FT of $r(\mathbf{r})$ is

$$
R\left(\mathbf{f}_{r}\right)=A \tilde{\delta}\left(\mathbf{f}_{r}\right)+\tilde{R}\left(\mathbf{f}_{r}-\mathbf{f}_{0}\right)+\tilde{R}^{*}\left(-\mathbf{f}_{r}-\mathbf{f}_{0}\right)
$$

where $\tilde{\delta}\left(\mathbf{f}_{r}\right)$ is Kronecker's delta function and $A$ is a constant that ensures a positive bias to make $r(\mathbf{r})$ real and positive. The FT of $r(\mathbf{r})$, given in Eq. (27), yields three terms. One of them, spatially separated from the others, is $\tilde{R}\left(\mathbf{f}_{r}\right)$, which is the FT of some function $\tilde{r}(\mathbf{r})$. This function is the desired bipolar or complex reference function. A similar procedure can be applied to the function $f(\mathbf{r})$ if it is complex too. If it is a real positive function, it is adequate to sample it at a spatial rate $\mathbf{f}_{0}$. This sampling is equivalent to a multiplication by the grating $0.5[1+$ $\left.\cos \left(k \mathbf{f}_{0} \cdot \mathbf{r}\right)\right]$, where $\mathbf{f}_{0}$ satisfies the condition $\left|\mathbf{f}_{0}\right| \geq$ $\max \left(W_{\tilde{R}} / 2, W_{G}\right)$. This sampling generates a first-order diffraction pattern of the form $G\left(\mathbf{f}_{r}\right)$ centered around point $\mathbf{f}_{0}$ in the $f_{r}$ domain. Centering the camera on the same point and recording only the first diffraction order results in the recorded distribution [see also Eq. (18)]

$$
\begin{aligned}
I_{2}{ }^{\prime}\left(\mathbf{f}_{r}\right)= & \operatorname{rect}\left(\frac{\mathbf{f}_{r}}{W_{\tilde{R}}}\right)\left[2 I_{\alpha}+2\left[\frac{4 f_{1}}{f_{2}^{2}}\right] \tilde{G}\left(\mathbf{f}_{r}+\mathbf{f}_{0}\right)\right. \\
& \left.+2\left[-\frac{4 f_{1}}{f_{2}^{2}}\right] \tilde{G}^{*}\left(\mathbf{f}_{r}+\mathbf{f}_{0}\right)\right] \\
= & 2 I_{\alpha}+2\left[\frac{4 f_{1}}{f_{2}^{2}}\right] U\left(\mathbf{f}_{r}\right)+2\left[-\frac{4 f_{1}}{f_{2}^{2}}\right] U^{*}\left(\mathbf{f}_{r}\right),
\end{aligned}
$$

where

$$
U\left(\mathbf{f}_{r}\right)=\tilde{R}\left(\mathbf{f}_{r}\right) \exp \left(j 2 \pi \mathbf{f}_{r} \cdot \mathbf{r}_{e}\right)+F\left(\mathbf{f}_{r}\right) \exp \left(-j 2 \pi \mathbf{f}_{r} \cdot \mathbf{r}_{e}\right) .
$$

Proceeding from Eq. (28) in steps similar to those taken from Eq. (18) to Eqs. (21) and (26), we obtain the correlation distribution $\tilde{r}(\mathbf{r}) * g(\mathbf{r})$ around the points $\pm \mathbf{r}_{e}$ in the JTC output plane ( $\mathrm{P}_{4}$ in Fig. 4). Hence the correlation between the object function and the complex reference function is obtained.

\section{INCOHERENT AND COHERENT SPATIAL FILTERING}

The general architecture of Fig. 4 is also suitable for a new kind of spatial filtering scheme. This procedure is based on the multiplication of the incoherent Fourier hologram [relation (10)] by a filter and the performance of a coherent reconstruction.

The output distribution of this system can be obtained by substituting the proper parameters into Eq. (15). The illumination of the input mask is completely incoherent; therefore $\gamma(\Delta \mathbf{r})=\delta(\Delta \mathbf{r})$. The angle between the planes is $180^{\circ}$, and we have a single input mask, i.e., $g_{1}(\mathbf{r})=$ $g_{2}(-\mathbf{r})=g(\mathbf{r})$. In the spatial frequency plane the original intensity distribution is multiplied by a filter $H\left(\mathbf{f}_{r}\right)$, and it is assumed that $C_{i}\left(\mathbf{f}_{r}\right)=\mathscr{L}\left[4 \mathbf{d}_{g} / f_{2}\right]$. Therefore Eq. (15) becomes

$$
\begin{aligned}
c(\overline{\mathbf{r}})= & h(\overline{\mathbf{r}}) \star\left[2 \delta(\overline{\mathbf{r}})+|g(\overline{\mathbf{r}})|^{2} \star \delta\left(\overline{\mathbf{r}}-\mathbf{d}_{g}\right)\right. \\
& \left.+|g(-\overline{\mathbf{r}})|^{2} \star \delta\left(\overline{\mathbf{r}}+\mathbf{d}_{g}\right)\right]
\end{aligned}
$$

where $h(\mathbf{r})$ is the inverse FT of $H\left(\mathbf{f}_{r}\right)$.

In principle, $H\left(\mathbf{f}_{r}\right)$ could be any complex function, but, as is the case in conventional coherent spatial filtering, the implementation of such a function is quite difficult. In this study we demonstrate the performance of the system by using holographic encoding, as in Ref. 18 for coherent illumination. A phase-only holographic filter ${ }^{19}$ to recognize a positive object function, $|f(\mathbf{r})|^{2}$, in a pattern recognition system is recorded in the FI as follows: Centering the function $f(-\mathbf{r})$ at a distance $\mathbf{d}_{f}$ from the optical axis in the input plane of the FI (Fig. 4), we obtain an intensity distribution in the spatial frequency domain similar to $I_{\mathscr{L}}\left(\mathbf{f}_{r}\right)$ of Eq. (23):

$$
I_{h}\left(\mathbf{f}_{r}\right)=2 I_{\alpha}+\mathscr{L}\left[\frac{4 \mathbf{d}_{f}}{f_{2}}\right] \tilde{F}^{*}\left(2 \mathbf{f}_{r}\right)+\mathscr{L}\left[-\frac{4 \mathbf{d}_{f}}{f_{2}}\right] \tilde{F}\left(2 \mathbf{f}_{r}\right),
$$

where $\tilde{F}\left(\mathbf{f}_{r}\right)$ is the FT of $|f(\mathbf{r})|^{2} . \quad I_{h}\left(\mathbf{f}_{r}\right)$ is processed, electronically, according to the following threshold rule:

$$
H\left(\mathbf{f}_{r}\right)=\left\{\begin{aligned}
1 & \text { if } I_{h}\left(\mathbf{f}_{r}\right) \geq 2 I_{\alpha} \\
-1 & \text { otherwise }
\end{aligned}\right.
$$

Thus $H\left(\mathbf{f}_{r}\right)$ is a binary hologram that may be expanded in a Fourier series as

$$
H\left(\mathbf{f}_{r}\right)=\sum_{n=-\infty, \text { odd }}^{n=\infty} \frac{2}{n \pi}(-1)^{(n-1) / 2} \exp \left\{j n\left[2 \pi \mathbf{f}_{r} \cdot \mathbf{d}_{f}+\varphi_{F}\left(\mathbf{f}_{r}\right)\right]\right\},
$$

where $\exp \left[j \varphi_{F}\left(\mathbf{f}_{r}\right)\right]$ is the phase distribution of $\tilde{F}\left(\mathbf{f}_{r}\right)$.

Placing this binary hologram at plane $\mathrm{P}_{2}$ of the system in Fig. 4 results in the required correlation, as in Eq. (30). However, this time $h(\tilde{\mathbf{r}})$ is obtained by an inverse FT of $H\left(\mathbf{f}_{r}\right)$ from Eq. (33), which is a superposition of many spatially separated terms. To avoid overlap among undesired cross-correlation terms, we ensure that the displacement $\mathbf{d}_{f}$ is perpendicular to $\mathbf{d}_{g}$, the displacement of the input function $|g(\mathbf{r})|^{2}$. The desired correlation distribution is obtained from the $(1,1)$ or the $(-1,-1)$ diffraction order around the point determined by the vector $\mathbf{d}_{h}+\mathbf{d}_{f}$ and given by

$$
s(\overline{\mathbf{r}})=\mathscr{F}\left\{\exp \left[j \phi_{F}\left(\mathbf{f}_{r}\right)\right]\right\} *|g(\overline{\mathbf{r}})|^{2} .
$$

When the input $g(\mathbf{r})$ contains objects of the form $f(\mathbf{r})$, the output distribution is characterized by narrow peaks located at the corresponding positions. ${ }^{19}$

In our experimental investigation we used the input distribution shown in Fig. 8(a). In the first experiment the filter was prepared to identify the letter T. Therefore 


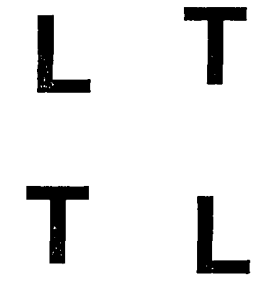

(a)

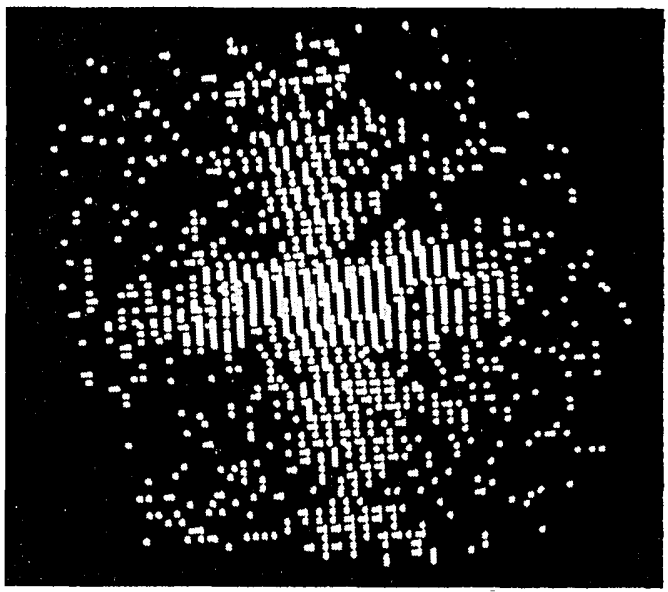

(b)

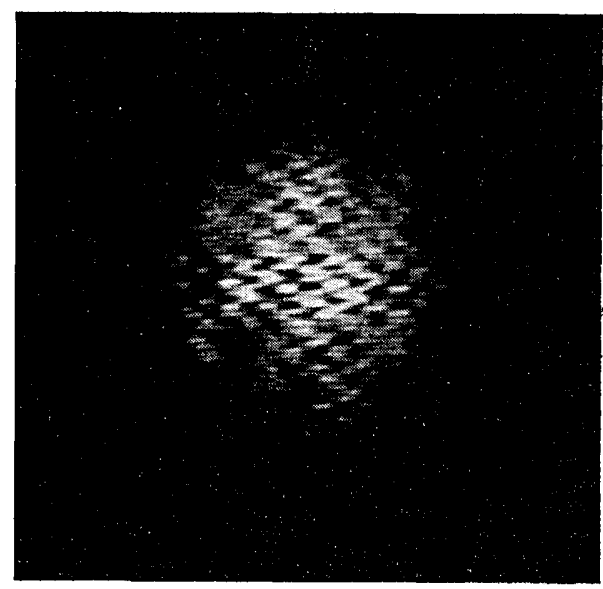

(c)

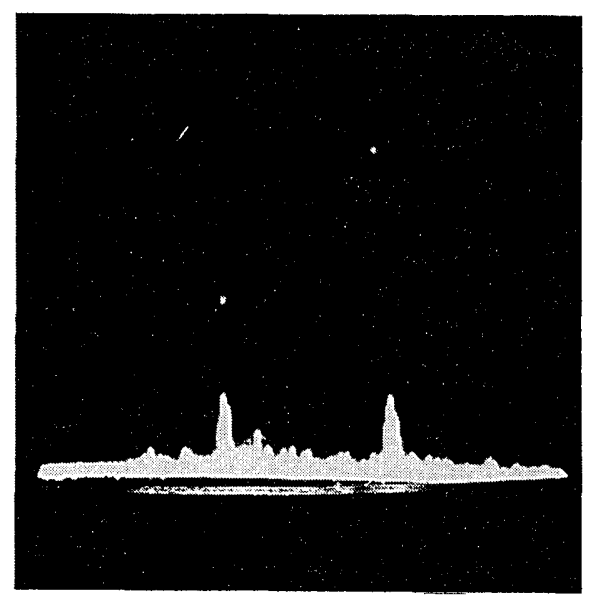

(d)

Fig. 8. (a) Input mask to the incoherent correlator. (b) Binary hologram used as the phase-only filter of the letter T. (c) Intensity distribution as recorded in plane $\mathrm{P}_{2}$ of Fig. 4 with the input mask of (a). (d) Correlation results of the incoherent correlator with a cross section from right to left through the upper peak.

this letter was first situated at point $\left(x_{f}, 0\right)$ of the FI input plane. The binary filter $H\left(\mathbf{f}_{r}\right)$, which was obtained according to the rule of Eq. (32), is presented in Fig. 8(b). This filter is multiplied by the spectrogram of the four letters that is shown in Fig. 8(c), and the product is transformed by the coherent FT system. The output crosscorrelation results are presented in Fig. 8(d).

\section{CONCLUSIONS}

In this paper we introduced a class of electro-optical signal processors that are capable of performing various operations by using coherent as well as spatially incoherent illumination. In the most general case three independent complex functions are involved in a degenerate case of triple-correlation processing [Eq. (16)]. One of the functions is always the normalized coherence function obtained by a FT of the source intensity. Many of the existing image processors can be shown to be special cases of this general class, of which we demonstrated two new incoherent correlators, for pattern recognition applications. Although they are based on interferometric architectures, these incoherent processors are not hindered by interferometric sensitivity to environmental noise and may offer new directions in optical image processing.

\section{ACKNOWLEDGMENTS}

The research of J. Rosen and J. Shamir was performed within the Technion Advanced Opto-Electronics Center established by the American Technion SocietyNew York. That of M. Segev and A. Yariv was supported by the U.S. Army Office of Scientific Research (Durham, N.C.).

\section{REFERENCES}

1. L. Mertz, Transformations in Optics (Wiley, New York, 1965).

2. K. V. Konjaev, "Interference method of two-dimensional Fourier transform with spatially incoherent illumination," Phys. Lett. A 24, 490-491 (1967).

3. S. Wang and N. George, "Fresnel zone transforms in spatially incoherent illumination," Appl. Opt. 24, 842-850 (1985).

4. E. Ribak, C. Roddier, F. Roddier, and J. B. Breckinridge, "Signal-to-noise limitations in white light holography," Appl. Opt. 27, 1183-1186 (1988).

5. J. B. Breckinridge, E. Ribak, C. Roddier, F. Roddier, and C. Habecker, "Real time optical correlation using white light Fourier transforms," final report of the technical work (Jet Propulsion Laboratory, California Institute of Technology, Pasadena, Calif., 1988).

6. R. N. Bracewell, H. Bartelt, A. W. Lohmann, and N. Streibl, "Optical synthesis of the Hartley transform," Appl. Opt. 24, 1401-1402 (1985). 
7. J. Villasenor and R. N. Bracewell, "Optical phase obtained by analogue Hartley transformation," Nature (London) 330, 735-737 (1987)

8. T. Nomura, K. Itoh, and Y. Ichioka, "Hartley transformation for hybrid pattern matching," Appl. Opt. 29, 4345-4350 (1990).

9. C. S. Weaver and J. W. Goodman, "A technique for optically convolving two functions," Appl. Opt. 5, 1248-1249 (1966).

10. M. Born and E. Wolf, Principles of Optics (Pergamon, New York, 1980)

11. R. N. Bracewell, "Discrete Hartley transform," J. Opt. Soc. Am. 73, 1832-1835 (1983)

12. Y. Fainman, E. Lenz, and J. Shamir, "Contouring by phase conjugation," Appl. Opt. 20, 158-163 (1981).

13. M. Segev and A. Yariv, "Optical interferometry between image-bearing beams and their redirected phase conjugates," Opt. Lett. 17, 145-147 (1992).
14. M. Cronin-Golomb, B. Fischer, J. O. White, and A. Yariv, "Passive (self pump) phase conjugate mirror: theoretical and experimental investigation," Appl. Phys. Lett. 41, 689691 (1982).

15. O. I. Potaturkin, "Incoherent diffraction correlator with a holographic filter," Appl. Opt. 18, 4203-4205 (1979).

16. D. Mendlovic, E. Marom, and N. Konforti, "Complex reference functions in joint transform correlator," Opt. Lett. 15, 12241226 (1990).

17. U. Mahlab, J. Rosen, and J. Shamir, "Iterative generation of complex reference functions in joint transform correlator," Opt. Lett. 16, 330-332 (1991).

18. J. Rosen, T. Kotzer, and J. Shamir, "Optical implementation of phase extraction pattern recognition," Opt. Commun. 83, 10-14 (1991).

19. J. L. Horner and P. D. Gianino, "Phase-only matched filtering," Appl. Opt. 23, 812-816 (1984). 